\title{
The Role of Ionic Surfactants in Compression Dewatering of Alum Sludge
}

\author{
C. P. Chu, ${ }^{*}$ D. J. Lee, ${ }^{* 1}$ and Chihpin Huang $\dagger$ \\ *Department of Chemical Engineering, National Taiwan University, Taipei, Taiwan 10617; and †Institute of Environmental Engineering, \\ National Chiao Tung University, Hsinchu, Taiwan, 30039, Republic of China \\ Received January 16, 1998; accepted May 31, 1998
}

\begin{abstract}
This work has experimentally investigated the characteristics of filtration followed by consolidation dewatering of an alum sludge, with especial attention to the effects of adding ionic surfactants (SDS or CTAB). The filtration and consolidation stages at a pressure of $3000 \mathrm{psi}$ were discussed separately. The efficiency of filtration is enhanced in the presence of surfactant molecules; however, the cationic surfactant (CTAB) raises the consolidation rate while the anionic surfactant (SDS) retards it. A newly proposed rheological model has been employed for interpreting the consolidation data. CTAB would not alter markedly the moisture distribution in the sludge, but SDS does increase markedly the amount of the tightly bound moisture by diminishing the portion occupied by pore water. The possible role of surfactants in the sludge flocs is considered. B oth surfactants can be used as conditioning aids during the filtration stage. However, the applications of SDS to the consolidation stage are not encouraged. 1998 Academic Press
\end{abstract}

Key Words: consolidation; sludge; ionic surfactants; rheological model.

\section{INTRODUCTION}

The way to achieve better dewatering of sludge is of essential importance in water and wastewater treatment plants. After chemical conditioning, the sludge is usually dewatered by mechanical means, such as a filter press or vacuum filter. Surfactants often appear in a vast amount in sewage sludges (1). The possible interactions between surfactant and polymer may lead to an excess polymer dose to achieve the same dewatering performance of sludge.

Surfactant molecules may be absorbed onto the solid's surface in the following six ways before its concentration exceeds the critical micelle concentration (CMC): (a) ion exchange, (b) ion pairing, (c) acid-base interaction (hydrogen-bonding), (d) adsorption by polarization of $\pi$ electrons, (e) adsorption by dispersion force (Van der Waals force), and (f) hydrophobic bonding (2). The characteristics of particle surface and dewaterability would definitely alter according to the adsorption of

\footnotetext{
${ }^{1}$ To whom correspondence should be addressed. E-mail: djlee@ccms. ntu.edu.tw.
}

surfactant molecules. However, information about the effects of ionic surfactants on the dewatering performance of sludge is largely lacking.

Compression is widely employed in industries to separate liquid from a cake by mechanical pressure (3). In practice, first the sludge is filtered to form a filtration cake, which is then directly consolidated by a membrane or a piston for further dewatering. Shirato and co-workers (4-10) and Tiller (3) made substantial contributions toward the understanding of constantpressure consolidation. A brief review is available in $(11,12)$. Knowledge about the underlying mechanisms for filtration followed by consolidation dewatering of sludges, nevertheless, has been still far from satisfactory.

Shirato et al. (7) adopted the combined Terzaghi-Voigt rheological model for describing the consolidation stage, and the cake thickness was obtained in the following manner:

$$
\begin{aligned}
U_{\mathrm{c}}=\frac{L_{1}-L}{L_{1}-L_{\mathrm{f}}}=(1-B)[1-\exp ( & \left.\left.-\frac{\pi^{2} i^{2} C_{\mathrm{e}}}{4 \omega_{0}^{2}} \theta_{\mathrm{c}}\right)\right] \\
& +B\left[1-\exp \left(-\eta \theta_{\mathrm{c}}\right)\right] .
\end{aligned}
$$

In Eq. [1], $U_{\mathrm{c}}$ is the consolidation ratio, $L$ the cake thickness, $L_{1}$ and $L_{\mathrm{f}}$, respectively, the initial and final cake thickness, $B$ the fraction of moisture removed by the secondary consolidation, $C_{\mathrm{e}}$ a parameter corresponding to the cake resistance to fluid flow, $i$ the number of drainage, $\omega_{0}$ the specific cake volume, $\theta_{\mathrm{c}}$ the consolidation time, and $\eta$ the creep factor, demonstrating the easiness of the relative mobility of constituting particles. Equation [1] is claimed to cover the whole consolidation process except for a short period after startup. Notably, inasmuch as the first exponential term in the righthand side of Eq. [1] usually decays much faster than the second one, at a large consolidation time, an asymptotic form can be obtained as in the following:

$$
U_{\mathrm{c}}=1-B \exp \left(-\eta \theta_{\mathrm{c}}\right)
$$

Restated, a linear $\ln \left(1-U_{\mathrm{c}}\right)$ versus $\theta_{\mathrm{c}}$ relationship holds at the final stage of consolidation. Some works had verified the 
applicability of Eq. [1] to certain sludges, such as some particulate slurries (8-10), a polymer flocculated sludge (13), an alum coagulated sludge (14), and a mixed sludge (15). Valuable information on the interactions between conditioning agents (polymer or alum) with the sludge particles was extracted from these studies.

We had discussed in this paper the effects of cationic or anionic surfactants on the filtration followed by consolidation characteristics of an alum sludge. The filtration data were analyzed through classical theory, while the consolidation data were interpreted on the basis of the Terzaghi-Voigt model (Eq. [1]) and a newly proposed rheological model (16) (discussed latter). According to the experimental results, the role of surfactant molecules in the sludge have been speculated.

\section{EXPERIMENTAL}

\section{Samples}

A sludge sample was taken from the sediment basins in the Men-Der water treatment plant. Its raw water comes directly from the nearby reservoirs, which are in serious eutrophication condition. A vast amount of algae is present in the plant as well as in the sludge. The chemicals applied in the plant include potassium permanganate as an algaecide and aluminum sulfate as a coagulant. The solid content of the sludge is approximately $10 \%$ (VSS is $2200 \mu \mathrm{g} \mathrm{C/g}$ ) and the sludge appears gray-black in color and is somewhat odorous.

The $\mathrm{pH}$ values for the alum sludge ranged from 7.2-7.6, which were adjusted to 8.0 for the sake of consistency among samples. At this $\mathrm{pH}$ the zeta potential of sludge was maintained around $-15 \mathrm{mV}$.

Two surfactants, anionic dodecylsulfate sodium salt (SDS) and cationic cetyltrimethyl-ammonium bromide (CTAB) were used. The mass weights of SDS and CTAB are $288.38 \mathrm{~g} / \mathrm{mol}$ and $364.45 \mathrm{~g} / \mathrm{mol}$, respectively. The surfactants with dosages far below their CMC (critical micelle concentration) were applied and completely mixed with sludge prior to conducting the compression test. The addition of SDS yields a more negative charge, while the CTAB leads to a less negatively charged surface.

In theory, most of soluble $\mathrm{Al}(\mathrm{III})$ is in the form of $\mathrm{Al}(\mathrm{OH})_{4}^{-}$ at $\mathrm{pH}$ 8. The influence of $\mathrm{Al}^{3+}$ or $\mathrm{Al}(\mathrm{OH})^{2+}$ can, therefore, be neglected. Other major cationic ions in the liquid phase of the sludge were $\mathrm{Ca}^{2+}$ and $\mathrm{Mg}^{2+}$. Their concentrations ranged from $20-30 \mathrm{mg} / \mathrm{L}$ and 5-10 $\mathrm{mg} / \mathrm{L}$, respectively. These ranges were very normal for natural waters. The presence of cationic ions should therefore have no significant effect on the surfactant's efficiency.

\section{Compression Test}

A constant head piston press (Triton Electronics Ltd., type 147) was employed in all tests. A schematic of the experimental setup can be found elsewhere $(13,14)$. The sludge was

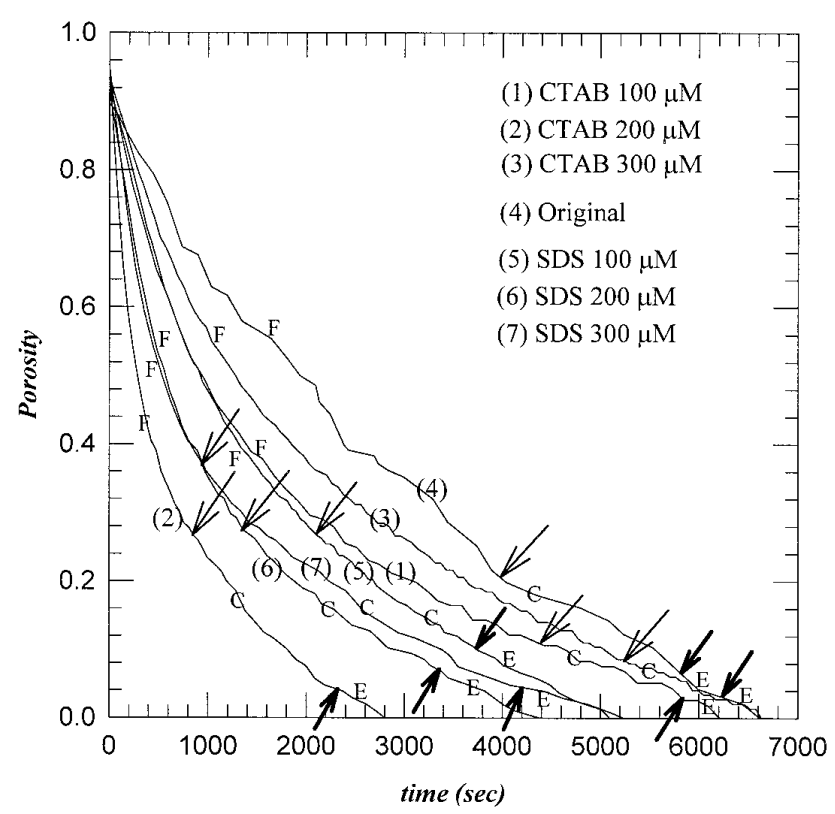

FIG. 1. Cake porosity versus time plot. $P=3000$ psi. F, filtration stage; $\mathrm{C}$, consolidation stage; E, final constant-rate period. Arrows indicate the transition points among stages.

placed in a stainless steel cylinder of diameter $7.62 \mathrm{~cm}$ and of height $20 \mathrm{~cm}$ equipped with a free piston. The cylinder is coated with chrome, and at one end there is a port. The high-pressure fluid with a hydraulic pressure of 3000 psi was exerted through the port onto the free piston, which pressed directly the sludge to force the moisture out. An electronic balance connected to a personal computer automatically recorded the time evolution of the filtrate weight. With the filtrate weight versus time data and the true solid density, the time evolution of cake porosity can be subsequently obtained.

\section{RESULTS}

Figure 1 depicts typical results for the time evolution of the cake porosity change. It is evident that both surfactants have positive effects on the overall filterability of sludge but complicated correlations with the dosages.

\section{Filtration Stage}

A complete test contains a filtration stage and a consolidation stage. The transition point is the moment when the piston has just touched the sludge cake, and it can be determined by means of the graphical method proposed by Shirato et al. (6). The filtration stage data can thereby be differentiated from the overall data set. The average specific resistance $\left(\alpha_{\text {av }}\right)$ of the sludge filtered at a constant pressure of 3000 psi can be calculated through following procedures. First the filtrate volume versus time relationship was constructed. Next the bestfitting slope of $t-V^{2}$ was substituted into the equation for estimating $\alpha_{\mathrm{av}}$, 


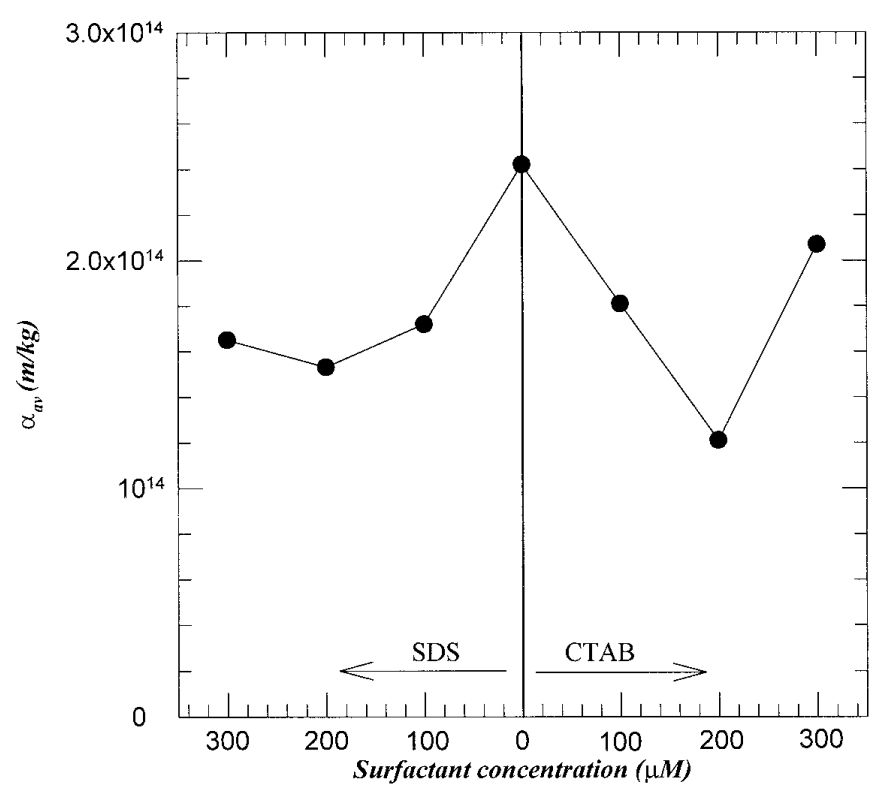

FIG. 2. The $\alpha_{\mathrm{av}}$ versus surfactant concentrations.

$$
\alpha_{\mathrm{av}}=\frac{2 P A^{2}(1-m s)}{\rho s \mu}\left(\frac{d t}{d V^{2}}\right)
$$

where $A$ is the filter area, $s$ the weight fraction of the solid, $m$ the weight ratio of final filter cake to total dry solid in the sludge, and $\rho$ and $\mu$ are the density and viscosity of filtrate, respectively. Other experimental procedures were summarized in (17). We herein neglect the contribution of bound water to the solid fraction counted in the sludge.

Figure 2 depicts the results for $\alpha_{\mathrm{av}}$. This figure indicates that both SDS and CTAB could reduce the average specific resistance of sludge. Restated, the presence of surfactant molecules would efficiently enhance the passage of fluid through the filter cake. Therefore, the surfactant addition is an aid to sludge dewatering during the filtration stage. It is noted as well that the excess surfactant dosage gains no further advantages. Figure 3 depicts the cake volume at the cease of filtration. The cake volume increases with increasing SDS dosage. On the other hand, the interactions between CTAB molecules and the sludge flocs lead to greater variations in cake volume.

\section{Consolidation Stage}

Figure 4 depicts the data of the consolidation stage. As Fig. 4a reveals, in all tests an initial decrease in $U_{c}$ follows a linear $\ln \left(1-U_{c}\right)-\theta_{c}$ stage (dashed line), as predicted by the TerzaghiVoigt model. However, an apparent deviation from the linear relationship occurs in the final phase of consolidation regardless of the presence of surfactants. On a normal-normal plot of $U_{\mathrm{c}}-\theta_{\mathrm{c}}$ this consolidation stage reveals a linear characteristic (Fig. 4b). (Notably, only representative data are depicted in these figures for the sake of clarity.) Restated, a constant-rate (rather than a logarithmically decaying rate) consolidation pe- riod appears at the final stage of consolidation, which violates the prediction of the Terzaghi-Voigt model. Considering the overall efficiency of consolidation dewatering, with some data scattering, the addition of CTAB would enhance compression dewatering, while SDS retards it. This is in contrast to the filtration stage where the surfactants could only enhance dewatering regardless of the sign of the surfactant's charge.

Figures 5 and 6 illustrate the best-fitting coefficients $B$ and $\eta$ on the basis of Eq.[1]. The Terzaghi-Voigt model assumes a visco-elastic sludge cake. Lower $B$ and greater $\eta$ values, that is, a less viscous and more elastic sludge cake, are favorable to dewatering (13-15). The $B$ values for sludges with or without surfactants are almost invariant (Fig. 5), indicating that the contribution of secondary consolidation is not affected by the presence of surfactants. However, the surfactant molecules do have an effect on the creep factor, $\eta$. According to the data depicted in Fig. 6, the creep factor decreases with addition of SDS, and increases when CTAB is added. Therefore, according to the conventional Terzaghi-Voigt model, the adsorption of cationic surfactant molecules onto the particle surface makes the relative movement of particles easier, while the anionic surfactant molecules have the opposite effect. The role of electrostatic repulsion may be therefore of significance.

Deviation from the conventional Terzaghi-Voigt model occurs in all tests depicted in Fig. 4 (the arrows). Kawasaki et al. (18) first noted the discrepancy from Eq. [1] at consolidating their biological sludge when $U_{\mathrm{c}}>0.8$. To extend Shirato's model for correcting the observed discrepancy, Chang and Lee (16) introduced the "ternary consolidation" stage, corresponding to the erosion of bound water in sludges. They had proposed a new rheological model that can properly describe the whole expression stage of an activated sludge. With the original sludge as a reference, as Fig. 4 reveals, the ternary con-

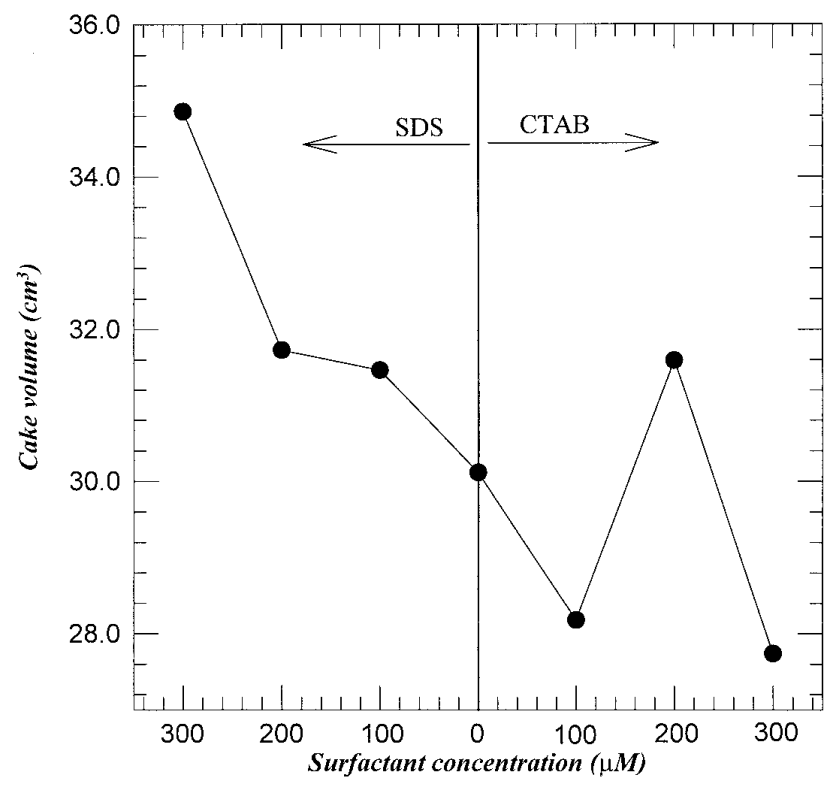

FIG. 3. Cake volume at the end of filtration. 
a

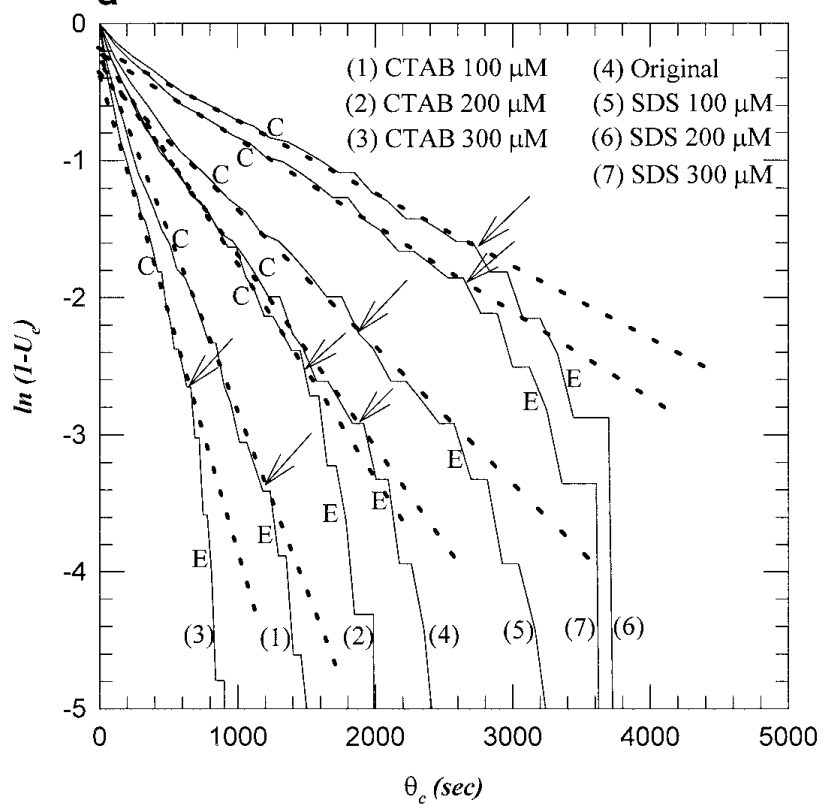

b

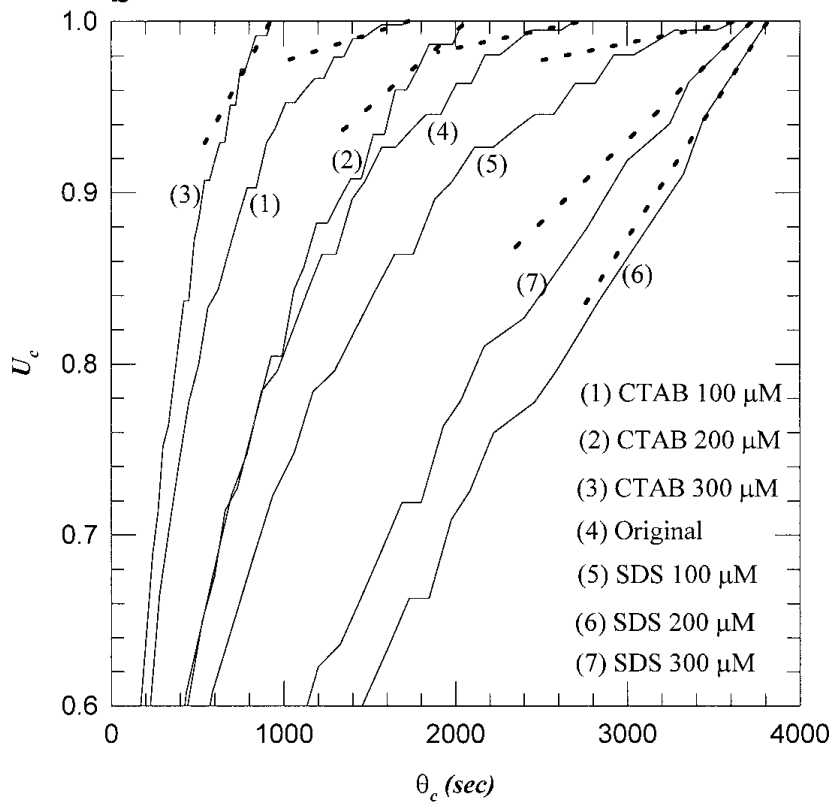

FIG. 4. (a) The $\ln \left(1-U_{\mathrm{c}}\right)$ versus $\theta_{\mathrm{c}}$ plot. $P=3000 \mathrm{psi}$; $\mathrm{C}$, consolidation period; $\mathrm{E}$, final constant-rate period. Arrows indicate the transition of the $\mathrm{E}$ region. Dashed lines are the regression line based on Eq. [1]. (b) The $U_{\mathrm{c}}$ versus $\theta_{\mathrm{c}}$ plot. Dashed lines indicate the final $U_{\mathrm{c}}-\theta_{\mathrm{c}}$ linear relationship.

solidation stage becomes more important with SDS. On the other hand, the CTAB has the opposite effect.

The modified model by Chang and Lee (16) is a three-stage model (Fig. 7) stated as

$$
\left(\frac{\partial e}{\partial \theta_{\mathrm{c}}}\right)_{\omega}=\left(\frac{\partial e}{\partial p_{\mathrm{s}}}\right)_{\theta \mathrm{c}}\left(\frac{\partial p_{\mathrm{s}}}{\partial \theta_{\mathrm{c}}}\right)_{\omega}+\left(\frac{\partial e}{\partial \theta_{\mathrm{c}}}\right)_{p_{\mathrm{s}}}^{(2)}+\left(\frac{\partial e}{\partial \theta_{\mathrm{c}}}\right)_{p_{\mathrm{s}}}^{(3)},
$$

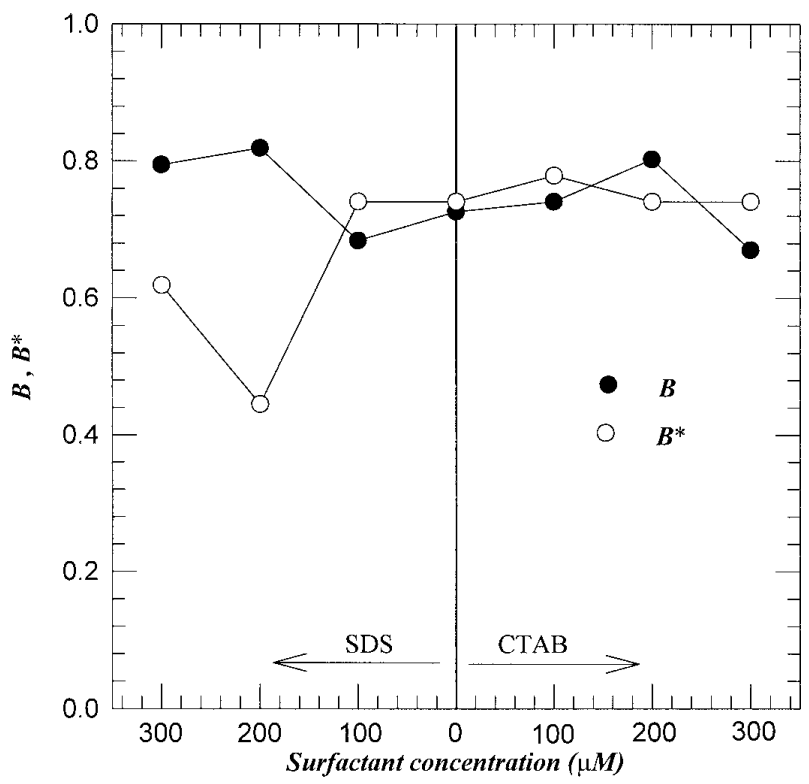

FIG. 5. The $B$ and $B^{*}$ versus surfactant concentrations. where $e, \omega, \theta_{\mathrm{c}}$, and $P_{\mathrm{s}}$ are the void ratio, specific volume of wet cake, consolidation time, and consolidation pressure, respectively. The first, second, and third term of the right-hand side of Eq. [4] represent, respectively, the primary, secondary, and ternary consolidation stages. The first two terms correspond to the conventional Terzaghi-Voigt model addressed in (7). We summarized herein briefly the derivation of the modified model for the sake of completeness.

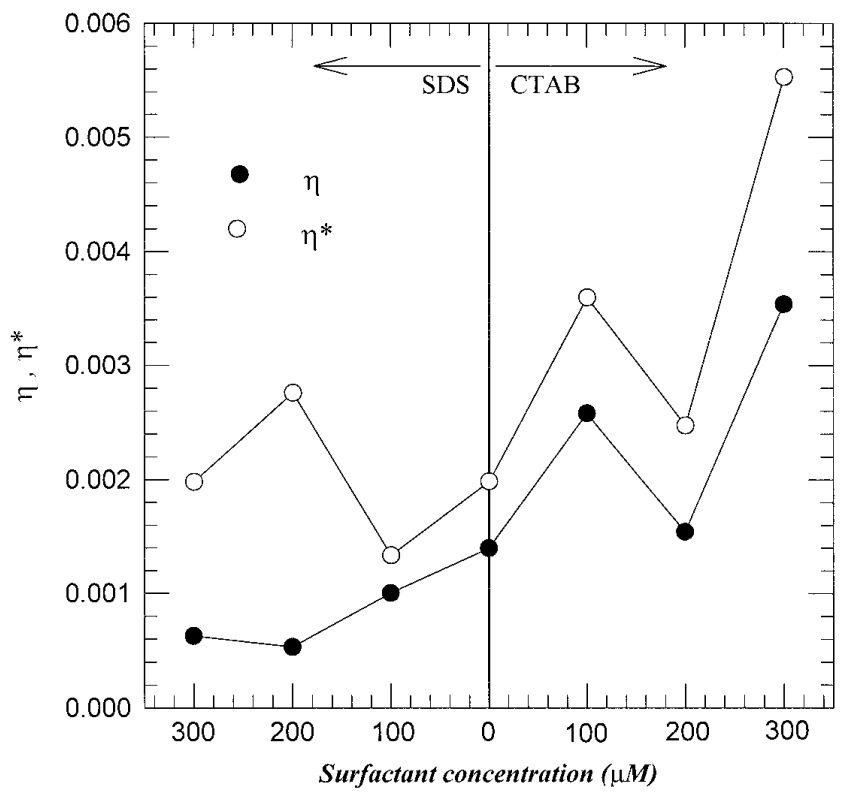

FIG. 6. The $\eta$ and $\eta^{*}$ versus surfactant concentrations. 


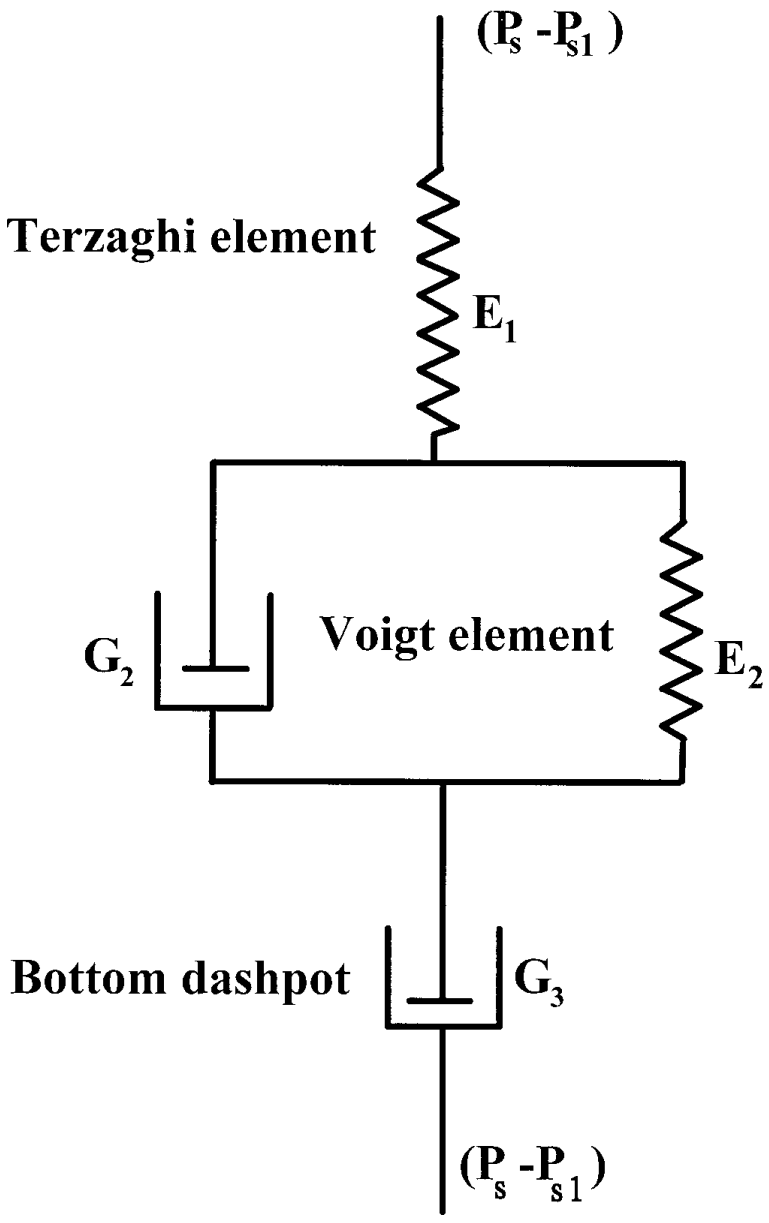

FIG. 7. Schematics of the rheological model adopted in (16).

The response of the third term, which is a dashpot of the coefficient of viscosity $G_{3}$, (highly viscous to model the erosion of bound water) is

$$
\left(\frac{\partial e}{\partial \theta_{\mathrm{c}}}\right)_{P_{\mathrm{s}}}^{(3)}=-\frac{\partial}{\partial \theta_{\mathrm{c}}}\left[a_{G}\left\{\int_{0}^{\theta_{\mathrm{c}}} P_{\mathrm{s}}(\omega, \tau) d \tau-\theta_{\mathrm{c}} P_{\mathrm{s} 1}\right\}\right],
$$

where $a_{G}=(1+e) / G_{3}$. Substituting Eq. [5] into Eq. [4], employing the Ruth-Sperry equation and the Leibnitz rule, leads to the partial differential equation

$$
\begin{aligned}
& \frac{\partial P_{\mathrm{s}}}{\partial \theta_{\mathrm{c}}}+(\beta \eta+\gamma) P_{\mathrm{s}}\left(\omega, \theta_{\mathrm{c}}\right)-\beta \eta^{2} \int_{0}^{\theta_{\mathrm{c}}} P_{\mathrm{s}}(\omega, \tau) \\
& \times \exp \left\{-\eta\left(\theta_{\mathrm{c}}-\tau\right)\right\} d \tau-\left\{\beta \eta \exp \left(-\eta \theta_{\mathrm{c}}\right)+\gamma\right\} P_{\mathrm{s} 1} \\
&=C e \frac{\partial^{2} P_{\mathrm{s}}}{\partial \omega^{2}},
\end{aligned}
$$

where $C_{\mathrm{e}}=\left(1 / \mu \rho_{\mathrm{s}} \alpha_{\mathrm{av}} a_{\mathrm{E}}\right), \alpha_{\mathrm{av}}$ is the average specific resis- tance of cake during consolidation, $\gamma$ is the ratio $a_{\mathrm{G}} / a_{\mathrm{E}}=$ $E_{1} / G_{3}, \beta$ is the ratio $a_{\mathrm{C}} / a_{\mathrm{E}}=E_{1} / E_{2}$, and $\mu$ and $\rho_{\mathrm{s}}$ are filtrate viscosity and solid phase density, respectively. Equation [6] has an analytical solution when considering the appropriate boundary and initial conditions. The cake thickness versus time relationship is found as follows except at a small consolidation time:

$$
\begin{aligned}
U_{\mathrm{c}}= & \frac{L_{1}-L}{L_{1}-L_{\mathrm{f}}} \\
= & (1-B-F)\left[1-\exp \left(-\frac{\pi^{2} i^{2} C e}{4 \omega_{0}^{2}} \theta_{\mathrm{c}}\right)\right] \\
& +B\left[1-\exp \left(-\eta \theta_{\mathrm{c}}\right)\right]+F\left(\frac{\theta_{\mathrm{c}}}{\theta_{\mathrm{c}}^{*}}\right) .
\end{aligned}
$$

The first and the second brackets in Eq. [7] account for the primary and the secondary consolidation stages as discussed in (7), while the third bracket is for the ternary consolidation. $(1-F-B)$ and $F$ are the fractions contributed by the primary and the ternary consolidation, respectively. $\theta_{\mathrm{c}}^{*}$ is the total consolidation time. At the $\theta_{\mathrm{c}} \rightarrow \infty$ limit, Eq. [7] becomes

$$
U_{3}^{(3)}=(1-F)+F \theta_{\mathrm{c}} / \theta_{\mathrm{c}}^{*}
$$

which assumes the final phase as a constant-rate compression. With an intermediate compression time, Eq. [7] becomes

$$
U_{\mathrm{c}}=(1-F)-B^{*} \exp \left(-\eta^{*} \theta_{\mathrm{c}}\right)
$$

which is a form similar to, and will reduce to, Eq. [2] if $F$ is small $(\gamma \rightarrow 0)$. The parameters $B^{*}, F$, and $\eta^{*}$ can be evaluated via regression analysis of different stages of data on the basis of Eqs. [8]-[9].

Table 1 lists the $\theta_{\mathrm{c}}^{*}$ and regressed $F$ values in accordance with Eq. [8]. (Notably, an apparent $\theta_{\mathrm{c}}^{*}$ was easily identified in experiments inasmuch as after this specific consolidation time the filtrate suddenly ceases to flow out from the sludge body.) The trend is approximately consistent with the arrows depicted in Fig. 4. Figure 8 depicts the $\ln \left(1-F-U_{c}\right)-\theta_{c}$ plot. A satisfactory linearity is observed for the intermediate period of the experimental data, thereby supporting the validity of Eq. [9]. The best-fitted $B^{*}$ and $\eta^{*}$ values are also plotted in Figs. 5 and 6. (Note, an asterisk is used to differentiate from the parameters on the basis of Eq. [2].)

As noted in Fig. 5, the $B^{*}$ values nearly coincide with $B$ for $\mathrm{CTAB}$, but show certain deviation for SDS. Restated, Eq. [2] would thereby give a fairly good estimate of $B$ for CTABcontaining sludge, but may be erroneous for SDS-containing sludge. This is attributed to the magnitude of the $F$ value estimated which makes a difference between Eqs. [2] and [9] (discussed later). 
TABLE 1

The Properties of the Consolidation Stage of Alum Sludge

\begin{tabular}{lccccc}
\hline & & & & & $\begin{array}{c}\text { Bound water } \\
\text { (kg/kg dry solid) }\end{array}$ \\
\hline Original & 0.17 & $B^{*}-F$ & & $\theta_{\mathrm{c}}^{*}(\mathrm{~s})$ & 2.81 \\
CTAB 100 $\mu M$ & 0.15 & 0.74 & 0.09 & 2407 & 2.24 \\
CTAB 200 $\mu M$ & 0.13 & 0.77 & 0.08 & 1494 & 2.79 \\
CTAB 300 $\mu M$ & 0.15 & 0.74 & 0.13 & 1991 & 2.53 \\
SDS 100 $\mu M$ & 0.18 & 0.74 & 0.11 & 906 & 3.29 \\
SDS 200 $\mu M$ & 0.00 & 0.55 & 0.08 & 3257 & 3.24 \\
SDS 300 $\mu M$ & 0.02 & 0.62 & 0.35 & 3740 & 3.11 \\
\hline
\end{tabular}

On the other hand, the newly found $\eta^{*}$ values are higher than those of Eq. [2]. Furthermore, the addition of SDS has almost no significant effect on $\eta^{*}$, in contrast to the conclusions drawn from the conventional Terzaghi-Voigt analysis. (Notably, the deviation in estimation of $\eta^{*}$ is greater than that of $\eta$, which is attributed to the possible error introduced in the estimate of parameter $F$. Although the error might be large, the trend for adding SDS is still different from that for CTAB.)

As a result, in this specific case, neglecting the ternary consolidation stage would introduce errors in estimating the creep factor, but give a fairly good estimate of the contribution of the secondary consolidation.

\section{DISCUSSIONS}

The surfactant addition is an aid to sludge dewatering during the filtration stage (Fig. 2). A lower resistance to filtration might be attributed to the larger aggregates' size, looser pack-

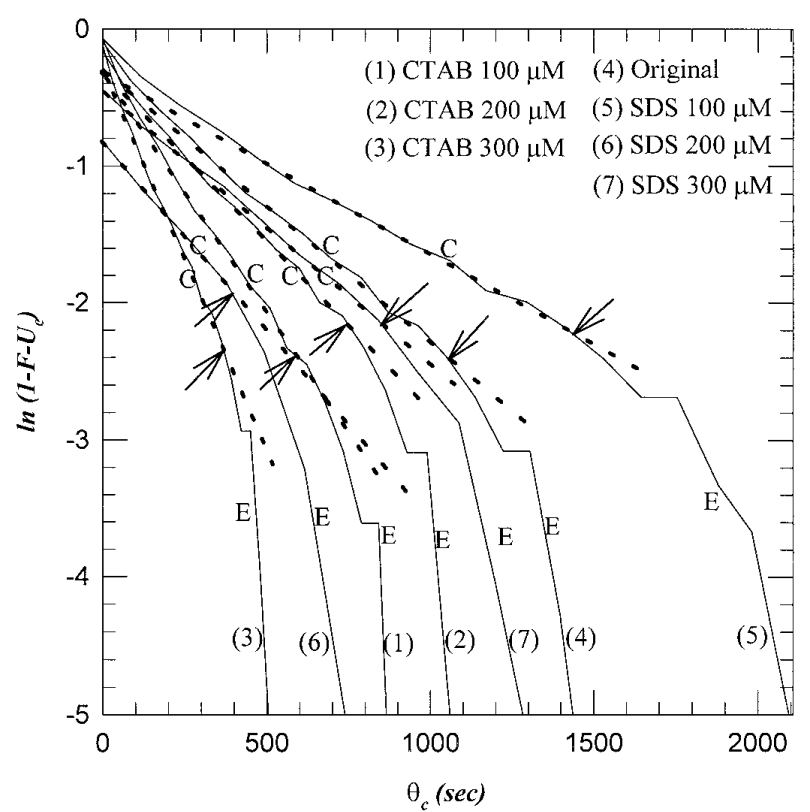

FIG. 8. The $\ln \left(1-F-U_{\mathrm{c}}\right)$ versus $\theta_{\mathrm{c}}$ plot. $\mathrm{C}$, consolidation period; E, final constant-rate period. Dashed lines are regression lines based on Eq. [7]. ing (higher porosity), less fine particle retention, less compressible cake structure, and other factors. As Fig. 3 reveals, the cake volume increases with increasing SDS dosage amount, which correlates well with the $\alpha_{\text {av }}$ data depicted in Fig. 2. The presence of SDS molecules can thereby yield a looser cake. However, the interaction between CTAB molecules and the sludge flocs are complicated, as well as the dependence on filtration resistance depicted in Fig. 2.

Dissipation of excess pore water and the collapse of cake global structure dominate the primary consolidation. A greater value of $\left(1-B^{*}-F\right)$ thereby denotes a weaker intra-aggregate strength. The secondary consolidation is usually interpreted as readjustment of constituent particles to a more stable configuration, whose rate is mainly controlled by shearing the highly viscous film of adsorbed water surrounding the particles' surfaces (19). A lower $\eta$ value (harder to creep) corresponds to a stronger inter-aggregate strength, while the greater $B$ value indicates a higher intra-aggregate strength (13-14). The mechanism of the ternary consolidation is proposed as the erosion of the tightly bound water from the sludge particle (16). Consequently, a greater $F$ value reveals a larger amount of tightly bound moisture in sludge, while an increasing $\theta_{\mathrm{c}}^{*}$ reflects the tighter binding of the moisture in the ternary consolidation stage.

As Figs. 5 and 6 reveal, the CTAB molecules have weakened the inter-aggregate strength (higher $\eta^{*}$ ), but have not affected the intra-aggregate strength (nearly constant $B^{*}$ ). On the other hand, the presence of SDS would not affect the inter-aggregate strength (nearly constant $\eta^{*}$ ), but has certain effects on the intra-aggregate strength (diminishing $B^{*}$ ). CTAB carries the opposite sign of charge to the sludge flocs. The adsorption of CTAB is electrostatically favorable, which would neutralize local charges. Without the net charge on the particle surface the passage of some particles across its neighboring particles would become easier (greater $\eta^{*}$ in Fig. 6) (13). The forces driving the adsorption of SDS should not be electrostatically related, but may have an origin in hydrophobic interaction, say. The more negative surface charge created would retard the passage of particles, thereby yielding a lower $\eta^{*}$ in Fig. 6. 
Table 1 also lists the relative moisture removal during primary, secondary, and ternary consolidation stages, $\left(1-B^{*}-\right.$ $\left.F, B^{*}, F\right)$. Apparently, the addition of CTAB has a negligible effect on the relative contributions of different consolidation stages. We herein had no clues for the decrease in filtration resistance. An easier aggregation of fine particles with flocs with lower surface charge may account for this.

However, the presence of SDS had significantly increased the contribution of the ternary consolidation $(F)$, by suppressing mainly the primary consolidation stage. Restated, SDS transforms some pore moisture into the tightly bound moisture in the sludge cake. The relatively more rigid cake structure for SDS-containing sludge cake (almost no primary consolidation exists) may correlate with the greater cake volume (Fig. 3) and the lower resistance to filtration (Fig. 2).

The total consolidation time $\theta_{\mathrm{c}}^{*}$ (listed in Table 1) decreases with CTAB but increases with SDS. The increase in $F$ value accompanies the greater total consolidation time.

Table 1 also lists the bound water contents of sludge. The definition of bound water in sludge is the remaining moisture in the cake after consolidation at 3000 psi (20). As Table 1 lists, the original and CTAB-containing sludge all exhibit a bound water of approximately $2.7 \mathrm{~kg} / \mathrm{kg}$ dry solid. It seems that the interactions between the CTAB molecules and the sludge flocs are limited to the outer layer of the aggregates, which reduces the surface charge and enhances creeping but affects the moisture distribution. On the other hand, the SDS molecules could bind more pore moisture onto the particle aggregates, along with which the elasticity of cake almost diminishes. The bound water content in SDS-containing sludge markedly increases. Together with the observation that SDS has almost no effect on the status of absorbed surface water (particle creeping), SDS molecules seem to interact mainly with the interior rather than the surface of the particle aggregates.

As a final note, the presence of CTAB is favorable for filtration and consolidation dewatering, and can therefore be employed as a conditioning aid. SDS, nevertheless, can be used to enhance filtration efficiency alone. The applications to the consolidation stage are not encouraged.

\section{CONCLUSIONS}

In this work the characteristics of filtration followed by consolidation dewatering and the water content of surfactantcontaining sludge were experimentally investigated at a pressure of 3000 psi. Both cationic (CTAB) and anionic (SDS) surfactants enhance the filtration efficiency; however, the former increases the consolidation rate while the latter retards it. A newly proposed rheological model by Chang and Lee (16) has been employed for interpreting the consolidation data. Errors can be introduced if the contribution of ternary consolidation stage is neglected, as it is in the conventional TerzaghiVoigt model. CTAB enhances particle creeping, but it does not alter the moisture distribution in the sludge. SDS increases significantly the amount of tightly bound moisture by diminishing the primary consolidation stage, but has significant effects on particle creeping. CTAB was speculated to interact mainly with the aggregates' surface while SDS, with the aggregates' interior. Both surfactants can be used as conditioning aids during the filtration stage. However, the applications of SDS to the consolidation stage are not encouraged.

\section{APPENDIX: NOMENCLATURE}

A Filter area, $\mathrm{m}^{2}$

$a_{\mathrm{C}} \quad$ Compressibility coefficient of secondary consolidation defined as $(1+e) / E_{2}, \mathrm{~Pa}^{-1}$

$a_{\mathrm{E}} \quad$ Compressibility coefficient of primary consolidation defined as $(1+e) / E_{1}, \mathrm{~Pa}^{-1}$

$a_{G} \quad$ Compressibility coefficient of ternary consolidation defined as $(1+e) / G_{3}, \mathrm{~Pa}^{-1} \mathrm{~s}^{-1}$

$B, B^{*}$ The ratio of secondary consolidation to the total consolidation,

$C_{\mathrm{e}} \quad$ Modified consolidation coefficient based on specific solid volume, $\mathrm{m}^{2} / \mathrm{s}$

$E_{1} \quad$ Rigidity of Terzaghi element, $\mathrm{Pa}$

$E_{2} \quad$ Rigidity of Voigt element, $\mathrm{Pa}$

$e^{\text {Local void ratio }}$

$F \quad$ The ratio of ternary consolidation to the total consolidation

$G_{2} \quad$ Viscosity of Voigt element of secondary consolidation, $\mathrm{Pa}^{-1}$

$G_{3} \quad$ Viscosity of Voigt element of ternary consolidation, $\mathrm{Pa}^{-1}$

$i \quad$ Number of drainage surface

$L \quad$ Cake thickness, $\mathrm{m}$

$L_{\mathrm{f}} \quad$ Final cake thickness, $\mathrm{m}$

$L_{1} \quad$ Initial cake thickness, $\mathrm{m}$

$m \quad$ Weight ratio of wet filter cake to dry one

$P \quad$ total consolidation pressure, $\mathrm{Pa}$

$P_{\mathrm{s}} \quad$ Local solid compressive pressure, $\mathrm{Pa}$

$P_{\mathrm{s} 1} \quad$ Local solid compressive pressure of material at beginning of consolidation, $\mathrm{Pa}$

$s$

Weight fraction of solid in the sludge

time, $s$

$U_{\text {c }} \quad$ Consolidation ratio

$V \quad$ Volume of filtrate, $\mathrm{m}^{3}$

$\alpha_{\text {av }} \quad$ Average specific resistance of cake, $\mathrm{m} / \mathrm{kg}$

$\beta \quad$ Parameter defined as $a_{\mathrm{C}} / a_{\mathrm{E}}$

$\gamma \quad$ Parameter defined as $a_{\mathrm{G}} / a_{\mathrm{E}}$

$\eta, \eta^{*} \quad$ Creep constant defined by $E_{2} / G_{2}, \mathrm{~s}^{-1}$

$\tau \quad$ Variable of Laplace transform, $\mathrm{s}$

$\theta_{\mathrm{c}} \quad$ Consolidation time, $\mathrm{s}$

$\theta_{\mathrm{c}}^{*} \quad$ Total consolidation time, $\mathrm{s}$

$\mu \quad$ Viscosity of filtrate, $\mathrm{Pa}-\mathrm{s}$

$\rho_{\mathrm{s}} \quad$ True density of solid, $\mathrm{kg} / \mathrm{m}^{3}$ 
$\rho \quad$ Density of filtrate, $\mathrm{kg} / \mathrm{m}^{3}$

$\omega \quad$ Variable indicating an arbitrary position in cake, $\mathrm{m}$

$\omega_{0} \quad$ Total solid volume in cake per unit of sectional area, $\mathrm{m}$

\section{ACKNOWLEDGMENTS}

This work is supported by the National Science Council, R.O.C. The authors appreciate Mr. C.G. Fu for his help in experimental works.

\section{REFERENCES}

1. Dentel, S. K. and Allen, H. E., "Proceedings, Wat. Envir. Fed. 70th Ann. Conf. Exposition, Chicago, Illinois, Oct. 18-22, 1997," p. 311, 1997.

2. Rosen, M. J., "Surfactants and Interfacial Phenomena," 2nd ed. Wiley, New York, 1989.

3. Tiller, F. M., and Yeh, C. S., AIChE J. 33, 1241 (1987).

4. Shirato, M., Murase, T., Kato, H., and Shibata, M., J. Fermentation Eng. 43, 255 (1965).

5. Shirato, M., Murase, T., Kato, H., and Fukaya, S., Kagaku Kogaku 31, 1125 (1965).

6. Shirato, M., Murase T., Kato, H., and Fukaya, S., Filtr. Sep. 7, 277 (1970).

7. Shirato, M., Murase, T., Tokunaga, A., and Yamada, O., J. Chem. Eng. Japan 7, 229 (1974).
8. Shirato, M., Murase T., Hayashi, N., and Fukushima, T., J. Chem. Eng. Japan 10, 154 (1977).

9. Shirato, M., Murase, T., Atsumi, K., Nagami, T., and Suzuki, H., J. Chem. Eng. Japan 11, 334 (1978).

10. Shirato, M., Murase, T., Atsumi, K., Aragaki, T., and Noguchi, T., J. Chem. Eng. Japan 12, 51 (1979).

11. Heij, E. J. La., Kerkhof, P. J. A. M., Herwijn, A. J. M., and Coumans, W. J., Water Res. 30, 697 (1996).

12. Chu, C. P., Chang, I. L., and Lee, D. J., Chem. Eng. 44, 79 (1997). [The Chin I. Ch. E., in Chinese].

13. Chang, I. L., Chu, C. P., Lee, D. J., and Huang C., J. Colloid Interface Sci. 185, 335 (1997).

14. Chang, I. L., Chu, C. P., Lee, D. J., and Huang, C., Environ. Sci. Technol. 31, 1313 (1997).

15. Chang, I. L., and Lee, D. J., Spectroscopy Lett. 29, 1659 (1996).

16. Chang, I. L., and Lee, D. J., Water Res. 32, 905 (1998).

17. Leu, W. F., Ph.D. Dissertation, University of Houston, Houston, Texas, 1981.

18. Kawasaki, K., Matsuda, A., and Murase, T., Int. Chem. Eng. 34, 403 (1994).

19. Craig, R. F., "Soil Mechanics," 5th ed., Chap. 7. Chapman \& Hall, London, 1993.

20. Chen, G. W., Hung, W. T., Chang, I. L., and Lee, D. J., J. Environ. Eng. ASCE. 123, 253 (1997). 